\title{
ON MOMENT INEQUALITIES AND STOCHASTIC ORDERING FOR WEIGHTED RELIABILITY MEASURES
}

\author{
BRODERICK O. OLUYEDE and MEKKI TERBECHE
}

(Received 15 March 2001)

\begin{abstract}
We obtain stochastic inequalities, error bounds, and classification probability for a general class of distributions. We introduce the notion of variability ordering via the probability functional and comparisons made for the weighted and the original distributions. We present moment inequalities, comparisons, and applications.
\end{abstract}

2000 Mathematics Subject Classification. 62N05, 62B10.

1. Introduction. Weighted distributions are of tremendous practical importance in various aspects of reliability, biometry, survival analysis and renewal theory to mention a few areas. In renewal theory the residual lifetime has a limiting distribution that is a weighted distribution with the weight function equal to the reciprocal of the hazard (failure) rate function. When observations are selected with probability proportional to their "length" the resulting distribution is referred to as a lengthbiased distribution. Length-biased distributions occur naturally in a wide variety of settings and are discussed by several authors including but not limited to Gupta and Akman [4], Zelen and Feinleib [6]. The problem of providing error bounds for exponential approximations to classes of life distributions in particular, the class of weighted distributions is addressed in this paper. Keilson [5] suggested a measure of departure from exponentiality within the class of completely monotone distributions (mixture of exponential distributions). These measures of departure are given in terms of $\rho=\left|\mu_{2} / 2 \mu^{2}-1\right|$, where $\mu_{2}=E\left(X^{2}\right)$ and $\mu=E(X)$. This is due to the fact that the exponential distribution satisfies $\rho=0$. Brown [2] obtained bounds for the class of increasing mean residual life (IMRL) functions.

The main objective of this paper is to obtain inequalities for weighted reliability measures, partial order via probability functional and compare reliability measures for weighted and in particular length-biased distributions. This paper is organized as follows. Section 2 contains some basic definitions, utility notions and comparisons. In Section 3 we present some moment inequalities for weighted reliability measures. In Section 4 some partial ordering via the probability functional are presented. The results are used to compare experiments for weighted distributions. Section 5 is concerned with comparisons for weighted and related distributions. The results are applied to length-biased mixtures of distributions.

2. Some definitions, utility notions, and comparisons. In this section, we present some definitions and useful notions. Let $\mathscr{F}$ be the set of absolutely continuous 
distribution function satisfying

$$
H(0)=0, \quad \lim _{x \rightarrow \infty} H(x)=1, \quad \sup \{x: H(x)<1\}=\infty .
$$

Note that if the mean of a random variable in $\mathscr{F}$ is finite, it is positive.

Let $X$ be a nonnegative random variable with reliability function $\bar{F}$ and probability density function (pdf) $f$, where $\bar{F}(x)=\int_{x}^{\infty} f(t) d t$. The weighted random variable has a pdf given by

$$
f_{W}(x)=\frac{W(x) f(x)}{\delta^{*}},
$$

where $\delta^{*}$ is a normalizing constant.

DEFINITION 2.1. A distribution function $F$ is said to have increasing mean residual life (IMRL) on $(0, \infty)$ if $\mu=\int_{0}^{\infty} \bar{F}(x) d x<\infty, F(0)<1$ and $E(X-x \mid X>x)$ is increasing in $x \geq 0$.

DEFINITION 2.2. A distribution function $F$ is said to have increasing (decreasing) hazard rate on $[0, \infty)$, denoted by IHR (DHR), if $F(0-)=0, F(0)<1$ and $P(X>x+t \mid$ $X>t)=\bar{F}(x+t) / \bar{F}(t)$ is decreasing (increasing) in $t \geq 0$ for each $x>0$.

Note that if $F$ has DHR and $\mu_{X}=\int_{0}^{\infty} \bar{F}(x) d x<\infty$, then $F$ has IMRL.

DEFINITION 2.3. Let $X$ and $Y$ be two nonnegative random variables with probability density functions $f$ and $g$, respectively. The random variable $X$ is said to be larger than $Y$ in monotone likelihood ratio ordering $\left(X \geq_{\operatorname{lr}} Y\right)$ if $f(x) / g(x)$ is nondecreasing in $x \geq 0$.

The following definition is due to Ebrahimi and Pellerey [3].

DEFINITION 2.4. The uncertainty of residual life distribution $H(X ; t)$, of a component at time $t$, is the entropy of the residual life random variable $(X-t \mid X>t)$, and is given by

$$
\begin{aligned}
H(X ; t) & =-\int_{t}^{\infty} \frac{f(x)}{\bar{F}(t)} \log \frac{f(x)}{\bar{F}(t)} d x \\
& =\log \bar{F}(t)-\{\bar{F}(t)\}^{-1} \int_{t}^{\infty} f(x) \log f(x) d x \\
& =1-\{\bar{F}(t)\}^{-1} \int_{t}^{\infty} f(x) \log \left\{\lambda_{F}(x)\right\} d x,
\end{aligned}
$$

where $\lambda_{F}(x)=f(x) / \bar{F}(x)$.

The entropy of the weighted residual life random variable $\left(X_{W}-t \mid X_{W}>t\right)$ is given by

$$
\begin{aligned}
H\left(X_{W} ; t\right) & =-\int_{t}^{\infty} \frac{f_{W}(x)}{\bar{F}_{W}(t)} \log \frac{f_{W}(x)}{\bar{F}_{W}(t)} d x \\
& =\log \bar{F}_{W}(t)-\left\{\bar{F}_{W}(t)\right\}^{-1} \int_{t}^{\infty} f_{W}(x) \log f_{W}(x) d x .
\end{aligned}
$$


Under length-biased sampling, $H\left(X_{W} ; t\right)$ reduces to

$$
H\left(X_{l} ; t\right)=1-\left\{\bar{F}(t) V_{F}(t)\right\}^{-1} \int_{t}^{\infty} x f(x) \log \left(\frac{x \lambda_{F}(x)}{V_{F}(x)}\right) d x
$$

where $V_{F}(t)=E(X-t \mid X>t)+t . H(X ; t)$ is the expected uncertainty in the conditional distribution of $X-t$, given $X>t$ about the predictability of the remaining lifetime of the component that has survived for time $t$.

Let $X_{t}$ denote the residual lifetime of a unit functioning at time $t$. Then as $t \rightarrow \infty$, $X_{t}$ has the limiting probability density function

$$
f_{e}(x)=\frac{\bar{F}(x)}{\mu_{F}}, \quad x \geq 0
$$

The length-biased equilibrium survival function is given by

$$
\bar{F}_{l_{e}}(x)=\left(\mu_{F}^{2}+\sigma_{F}^{2}\right)^{-1} \int_{x}^{\infty} \bar{F}(u)\left\{u+\delta_{F}(u)\right\} d u,
$$

where $\delta_{F}(u)=\int_{x}^{\infty} \bar{F}(t) d t / \bar{F}(u), u \geq 0$, and $\sigma_{F}^{2}$ is the variance of $F$. Note that if the weight function $W(x)$ is increasing on $[0, \infty)$, then $Y \geq_{\operatorname{lr}} X$ and $\lambda_{G}(x) \leq \lambda_{F}(x)$ for all $x \geq 0$, so that $\bar{G}(x) \geq \bar{F}(x)$ for all $x \geq 0$.

Proposition 2.5. If $X \leq_{\operatorname{lr}} Y$, then $H(X ; t) \leq H(Y ; t)$, for all $t \geq 0$, where $Y$ is the length-biased random variable with equilibrium distribution $F_{l_{e}}$.

Proof. The length-biased equilibrium survival function is given by

$$
\bar{F}_{l}(x)=\frac{\bar{F}(x) \delta_{F}(x)}{\mu_{X}},
$$

where $\delta_{F}(x)=V_{F}(x)-x$, and $\mu_{X}=\int_{0}^{\infty} \bar{F}(x) d x$. Clearly, $\bar{F}_{l}(x) \geq \bar{F}(x)$, for all $x \geq 0$. This follows from the fact that $Y \geq_{\operatorname{lr}} X$, so that $X$ has a DFR distribution. Consequently, $H(X ; t) \leq H(Y ; t)$, for all $t \geq 0$.

3. Moment inequalities for weighted reliability measures. In this section, we present some inequalities for weighted distributions. Let $\mathscr{F}$ be the set of absolutely continuous distribution function given by (2.1). The weighted survival function is given by

$$
\bar{G}(x)=\delta^{*-1} \int_{x}^{\infty} W(y) d F(y),
$$

where $W(y)$ is a positive real function and $0<\delta^{*}=E(W(X))<\infty$.

It is well known that if $G_{1}$ and $G_{2}$ are absolutely continuous with respect to a $\sigma$-finite measure $v$, with Radon-Nikodym derivative $g_{1}$ and $g_{2}$, then

$$
\int\left|g_{2}-g_{1}\right| d v=2 \sup _{\gamma}\left|G_{1}(\Delta)-G_{2}(\Delta)\right|
$$

where $\gamma$ is the collection of Borel subsets of $[0, \infty)$. Indeed if $P(X=Y)$ is small, then $g_{1}$ and $g_{2}$ are close in $L_{1}(v)$ norm, where the distributions of $X$ and $Y$ are given by 
$G_{1}$ and $G_{2}$, respectively. The weighted survival function $\bar{G}(x)$ can be written as

$$
\frac{\bar{G}(x)}{\bar{F}(x)}=\frac{E[W(X) \mid X>x]}{\delta^{*}}
$$

and the corresponding ratio of hazard functions is given by

$$
\frac{\lambda_{G}(x)}{\lambda_{F}(x)}=\frac{W(x)}{E[W(X) \mid X>x]}
$$

The quantity $E[W(X) \mid X>x]$ is referred to as the weighted vitality function. Under size-biased sampling, $W(x)=x$ and

$$
V_{F}(x)=E(X-x \mid X>x)+x,
$$

where

$$
E[X-x \mid X>x]=\int_{x}^{\infty} \frac{\bar{F}(y) d y}{\bar{F}(x)}
$$

is the residual life function (MRLF).

It is clear that if $V_{F}(x)$ is increasing in $x$, then

$$
\bar{G}_{l}(x)=\mu^{-1} \int_{x}^{\infty} y d F(y) \geq c^{-1} \bar{F}(x) \geq \bar{F}(x),
$$

where $c=\bar{F}(0), \bar{G}(0)=1$, and $c^{-1}=\bar{G}(0) / \bar{F}(0)$. Indeed if the weighted vitality function $E[W(X) \mid X>x]$ is increasing in $x$, then $\bar{G}(x) / \bar{F}(x)$ is increasing in $x$.

THEOREM 3.1 (see [1]). If $F$ has DMRL, then $S_{k}(x) \leq S_{k}(0) e^{-x / \mu}, k=1,2, \ldots$, and $S_{k}(x) \geq \mu S_{k-1}(0) e^{-x / \mu}-\mu S_{k-1}(0)+S_{k}(0), k=2,3, \ldots$, where

$$
S_{k}(x)= \begin{cases}\bar{F}(x) & \text { if } k=0, \\ \int_{0}^{\infty} \frac{\bar{F}(x+t) t^{k-1} d t}{(k-1) !} & \text { if } k=1,2, \ldots,\end{cases}
$$

is a sequence of decreasing functions for which $F$ possess moments of order $J$, that is, $\mu_{k}=E\left(X^{k}\right)$ exists, $k=1,2, \ldots, J$.

Let $S_{-1}(x)=f(x)$ be the pdf of $F$ if it exists. Then $S_{k}(0)=\mu_{k} / k !$, and $S_{k}^{\prime}(x)=$ $-S_{k-1}(x), k=0,1,2, \ldots, J$. The ratio $S_{k-1}(x) / S_{k}(x)$ is a hazard function of a distribution function with survival function $S_{k}(x) / S_{k}(0)$. The inequalities in Theorem 3.1 are reversed if $F$ has increasing mean residual life (IMRL).

THEOREM 3.2. Let $\bar{G}(x)$ be an IHR weighted distribution function with monotone weight function. Then

$$
\int_{0}^{\infty}\left|\bar{G}(x)-\bar{G}_{l}(x)\right| d x \leq 2 \mu(1+\beta),
$$

where $\beta=\mu-\mu_{2} / 2 \mu^{2}$, and $\bar{G}_{l}(x)=(1+x / \mu) e^{-x / \mu}$, for $x \geq 0$. 
Proof. Let $A=\{x \mid \bar{G} \leq(1+x / \mu) \exp (-x / \mu)\}$. Then we have for $x \geq 0$,

$$
\begin{aligned}
\int_{0}^{\infty} \mid & \bar{G}(x)-\left(1+\frac{x}{\mu}\right) e^{-x / \mu} \mid d x \\
& \leq 2 \int_{A}\left(\left(1+\frac{x}{\mu}\right) e^{-x / \mu}-\bar{G}(x)\right) d x \\
& \leq 2 \int_{0}^{\infty}\left(\left(1+\frac{x}{\mu}\right) e^{-x / \mu}-\bar{F}(x)\right) d x \\
& =2 \int_{0}^{\infty}\left(\left(1+\frac{x}{\mu}\right) e^{-x / \mu}-\frac{S_{1}(x)}{\mu}\right) d x=2\left(\mu^{2}+\mu-\frac{\mu_{2}}{2 \mu}\right) \\
& =2 \mu\left(1+\mu-\frac{\mu_{2}}{2 \mu}\right)=2 \mu(1+\beta) .
\end{aligned}
$$

The first inequality is trivial and the second inequality is due to the stochastic order between $\bar{G}(x)$ and $\bar{G}_{l}(x)$ whenever $W(x)$ is increasing in $x \geq 0$.

THEOREM 3.3. Let $\bar{G}(x)$ be a DHR weighted distribution function with monotone weight function $W(x) \geq 0$. Then for $\epsilon \geq \mu \geq 1$,

$$
\int_{0}^{\infty}\left|\bar{G}(x)-e^{-x / \mu}\right| d x \geq 2 \mu e^{-\epsilon / \mu}(\mu-1) .
$$

Proof. Let $\epsilon \geq \mu$, then for $x \geq 0$, we have

$$
\begin{aligned}
\int_{0}^{\infty}\left|\bar{G}(x)-e^{-x / \mu}\right| d x & =2 \int_{\epsilon}^{\infty}\left\{\bar{G}(x)-e^{-x / \mu}\right\} d x \\
& \geq 2 \int_{\epsilon}^{\infty}\left\{\bar{F}(x)-e^{-x / \mu}\right\} d x \\
& =2\left(\mu S_{1}(\epsilon)-\mu e^{-\epsilon / \mu}\right) \\
& \geq 2 \mu\left\{S_{1}(0) e^{-\epsilon / \mu}-e^{-\epsilon / \mu}\right\} \\
& =2 \mu e^{-\epsilon / \mu}\left(S_{1}(0)-1\right) \\
& =2 \mu e^{-\epsilon / \mu}(\mu-1) .
\end{aligned}
$$

The first inequality is due to the fact that $\bar{G}(x)$ and $\bar{F}(x)$ are stochastic ordered whenever $W(x)$ is increasing in $x \geq 0$, and the second inequality follows from Theorem 3.1.

The next result is due in part to an application of the lemma given by Brown [2].

THEOREM 3.4. Assume that the weighted vitality function $E[W(X) \mid X>x]$ is increasing in $x, 0<\delta^{*}=E[W(X)]<\infty$ and $\mu_{F}=\int_{0}^{\infty} \bar{F}(x) d x<\infty$, then

$$
\sup _{x}|\bar{G}(x)-\bar{F}(x)| \leq 1-\frac{\delta^{*}}{\mu_{G}}
$$

where $\mu_{G}=\int_{0}^{\infty} \bar{G}(x) d x$. 
Proof. Since $E[W(X) \mid X>x]=\delta^{*} \bar{G}(x) / \bar{F}(x)$ is increasing, we obtain

$$
\begin{aligned}
\sup _{x}|\bar{G}(x)-\bar{F}(x)| & \leq 1-\delta^{*-1} \int_{0}^{\infty}\left(\frac{\bar{F}(x)}{\bar{G}(x)}\right) W(x) f(x) d x \\
& =1-\delta^{*-1} \int_{0}^{\infty}\left(\frac{\bar{F}(x)}{\bar{G}(x)}\right)^{2}\left(\frac{\bar{F}(x)}{\bar{G}(x)}\right) W(x) f(x) d x \\
& \leq 1-\delta^{*-1} \mu_{G}\left(\int_{0}^{\infty} \frac{W(x) f(x) d x}{\mu_{G}}\right)^{2} \\
& =1-\frac{\delta^{*}}{\mu_{G}}
\end{aligned}
$$

THEOREM 3.5. Under length or size-biased sampling

$$
\sup _{x}\left|\bar{G}_{l}(x)-\bar{F}(x)\right| \leq 1-\frac{\mu^{2}}{\mu_{2}}=\frac{\sigma_{F}^{2}}{\left(\sigma_{F}^{2}+\mu_{F}^{2}\right)},
$$

where $\mu_{2}=E\left(X^{2}\right)$ and $\sigma_{F}^{2}=\operatorname{Var}(X)$ is the variance of $X$.

Proof. This follows from the fact that

$$
E_{G}\left(X^{r}\right)=\frac{E_{F}\left(X^{r+1}\right)}{\mu_{F}}
$$

$r \geq 1$ and $\delta^{*}$ reduces to $\mu_{F}=\int_{0}^{\infty} \bar{F}(x) d x$.

4. Partial ordering via probability functional. Let $(X, y)$ be a random vector, where $X$ lies on the real line and is observable. Let $y$ be 0 or 1 . We assume the conditional probability density function of $X$, given $y=1$ and $y=0$ are $g_{1}(x)=f(y \mid x=1)$ and $g_{0}(x)=f(y \mid x=0)$, respectively. Consider a simple classification procedure defined as a partition of $C=\left(C_{0}, C_{1}\right)$ in $\mathbb{R}$, such that $y_{1}=i$ if and only if $X \in C_{i}, i=0,1$. The mean probability of true classification is

$$
P\left(Y_{1}=y\right)=\frac{1}{2} P\left(X \in C_{0} \mid y=0\right)+\frac{1}{2} P\left(X \in C_{1} \mid y=1\right)
$$

For this setting, the optimal Bayes rule $A=\left(A_{0}, A_{1}\right)$ that maximizes the mean probability of true classification is of the form

$$
A_{0}=\left\{x \mid g_{0}(x) \geq g_{1}(x)\right\}, \quad A_{1}=\left\{x \mid g_{1}(x)>g_{0}(x)\right\}
$$

The true probability of classification is given by

$$
\Delta=\frac{1}{2} \int_{\mathbb{R}} \max \left(g_{0}(x), g_{1}(x)\right) d x=\frac{1}{2}+\frac{1}{4} \int_{\mathbb{R}}\left|g_{0}(x)-g_{1}(x)\right| d x .
$$


For the weighted distribution with weight function $W(x) \geq 0$ and $0<E[W(X)]<\infty$, the probability functional (4.3) reduces to

$$
\begin{aligned}
\frac{1}{2} \int_{0}^{\infty} \max \left(\frac{W(x) f(x)}{E(W(X))}, f(x)\right) d x \\
\quad=\frac{1}{2}+\frac{1}{4 \delta}\{E\{\max (W(X)-\delta, 0)\}+E\{\max (\delta-W(X), 0)\}\},
\end{aligned}
$$

where $0 \neq \delta=E[W(X)]$.

Let $g_{W_{i}}(x)=W_{i}(x) f_{i}(x) / \delta_{i}, i=1,2$, then

$$
\int_{0}^{\infty} \max \left(g_{W_{1}}(x), f_{1}(x)\right) d x \geq \int_{0}^{\infty} \max \left(g_{W_{2}}(x), f_{2}(x)\right) d x
$$

if and only if

$$
E\left|\left\{\frac{W_{1}(X)}{\delta_{1}}\right\}-1\right| \geq E\left|\left\{\frac{W_{2}(X)}{\delta_{2}}\right\}-1\right|,
$$

where $0 \neq \delta_{i}=E\left[W_{i}(X)\right], i=1,2$.

Let $\left(X_{i}, y_{i}\right)$ be random vectors and $g_{0 i}$ and $g_{1 i}, i=1,2$, be given by (4.3).

DEFINITION 4.1. We say the random vector $\left(X_{1}, y_{1}\right)$ is more varied than the random vector $\left(X_{2}, y_{2}\right)$ if

$$
\Delta_{1}=\frac{1}{2}+\frac{1}{4} \int_{\mathbb{R}}\left|g_{01}(x)-g_{11}(x)\right| d x \geq \Delta_{2}=\frac{1}{2}+\frac{1}{4} \int_{\mathbb{R}}\left|g_{02}(x)-g_{22}(x)\right| d x .
$$

This is denoted by $\left(X_{1}, y_{1}\right) \stackrel{\mathrm{mv}}{\geq}\left(X_{2}, y_{2}\right)$.

Now suppose one of the hypotheses given below is true. The hypotheses are

$$
\begin{array}{ll}
H_{0}:\left(X_{1} \mid y_{1}=0\right) \sim g_{0}, & \left(X_{1} \mid y_{1}=1\right) \sim g_{1}, \\
H_{1}:\left(X_{1} \mid y_{1}=0\right) \sim g_{1}, & \left(X_{1} \mid y_{1}=1\right) \sim g_{0} .
\end{array}
$$

Let $\pi_{i}$ denote the prior probability that $H_{i}$ is true and $R\left(\pi_{i}\right)$ the Bayes risk when the experiment $X_{1} \mid y_{1}=i, i=0,1$ is the case. The next theorem allows for the comparison of two experiments $X_{1} \mid y_{1}=1$ and $X_{1} \mid y_{1}=0$ in terms of Bayes risk.

\section{THEOREM 4.2.}

$$
R_{X_{1} \mid y_{1}=1}\left(\pi_{0}\right) \geq R_{X_{1} \mid y_{1}=0}\left(\pi_{0}\right)
$$

if and only if

$$
\int_{\mathbb{R}} \max \left(g_{0}(x), \gamma g_{1}(x)\right) d x \geq \int_{\mathbb{R}} \max \left(\gamma g_{0}(x), g_{1}(x)\right) d x,
$$

where $\gamma=\alpha_{0} \pi_{0} / \alpha_{1} \pi_{1}, \pi_{1}>0$ and $\alpha_{i}>0$ is the loss if $H_{i}$ is true and not accepted. 
Proof. Let $A=\left\{x: y g_{0}(x) \leq g_{1}(x)\right\}$ and $d_{i}$ the Bayes decision to accept $H_{i}$. Then

$$
\begin{aligned}
R_{X_{1} \mid y_{1}=1}\left(\pi_{0}\right) & =\alpha_{0} \pi_{0} P\left(d_{1} \mid H_{0}\right)+\alpha_{0} \pi_{0} P\left(d_{0} \mid H_{1}\right) \\
& =\alpha_{0} \pi_{0} \int_{A} g_{1}(x) d x+\alpha_{1} \pi_{1} \int_{A^{c}} g_{0}(x) d x \\
& =\alpha_{1} \pi_{1} \int_{\mathbb{R}} \max \left(\gamma g_{0}(x), g_{1}(x)\right) d x, \\
R_{X_{1} \mid y_{1}=1}\left(\pi_{0}\right) & =\alpha_{1} \pi_{1} \int_{\mathbb{R}} \max \left(g_{0}(x), \gamma g_{1}(x)\right) d x,
\end{aligned}
$$

where $\gamma=\alpha_{0} \pi_{0} / \alpha_{1} \pi_{1}, \pi_{1}>0$. The result now follows. If $\gamma=1$ or $g_{0}$ and $g_{1}$ are mutually disjoint, then $R_{X_{1} \mid y_{1}=1}\left(\pi_{0}\right)=R_{X_{1} \mid y_{1}=0}\left(\pi_{0}\right)$. The optimal choice of experiment depends only on $g_{0}, g_{1}$ and the value of $\gamma$.

5. Comparisons for weighted and related models. It is important to compare experiments for some family of models. For any such comparisons, interest should be in comparisons that are compatible and practically possible. In this light, one might be inclined to investigate and compare the possibility of sampling or selection of experiment from weighted distribution as opposed to the parent or original distribution. In a similar setting comparison might be restricted to a class of distributions including possibly distributions with monotone likelihood ratios, comparisons via some informational measures such as informational energy functions, Fisher, Shannon or Kullback-Leibler information.

Let the conditional density of $X$, given $y=1$, differ from $g_{0}(x)$ by a shift, that is, $g_{1}(x)=g_{0}(x-c)$. Let $H\left(g_{0}, c\right)=\int_{\mathbb{R}}\left|g_{0}(x)-g_{0}(x-c)\right| d x, c>0$.

\section{PROPOSITION 5.1.}

$$
\inf _{g_{0} \in G} H\left(g_{0}, c\right) \leq c(a)^{-1 / 2},
$$

where $G$ is the class of probability density functions on $\mathbb{R}$ with constant variance.

Proof. Consider the family of distribution functions with mean zero and finite support on the interval $[-\sqrt{a}, \sqrt{a}], a>0$, and the random variable $U$ such that $P(U=$ $-\sqrt{a})=P(U=\sqrt{a})=1 / 2$. Then

$$
E|U-c| \geq E|X-c|
$$

for all $c>0$ and by the assumption

$$
H\left(g_{0}, c\right)=c(a)^{-1 / 2} .
$$

Consequently,

$$
\inf _{g_{0} \in G} H\left(g_{0}, c\right) \leq c(a)^{-1 / 2}
$$


Let $f(x)$ and $f_{l}(x)$ denote the original and length-biased probability density functions, respectively. The true probability of classification in this regard is given by

$$
\begin{aligned}
\Delta_{l} & =\frac{1}{2} \int_{0}^{\infty} \max \left(f(x), f_{l}(x)\right) d x=\frac{1}{2}+\frac{1}{4} \int_{0}^{\infty}\left|f(x)-x f(x) / \mu_{X}\right| d x \\
& =\frac{1}{2}+\frac{1}{4 \mu_{X}} \int_{0}^{\infty}\left|X-\mu_{X}\right| d x=\frac{1}{2}+\frac{1}{4 \mu_{X}} E\left\{\left|X-\mu_{X}\right|\right\} .
\end{aligned}
$$

It is clear that

$$
\int_{0}^{\infty}\left|f(x)-f_{l}(x)\right| d x=\frac{1}{4 \mu_{X}} \int_{0}^{\infty}\left(1-F_{X-\mu_{X}}(u)\right) d u,
$$

due to the fact that

$$
\begin{aligned}
E\left|X-\mu_{X}\right| & =E\left\{\max \left(X-\mu_{X}, 0\right)\right\}+E\left\{\max \left(\mu_{X}-X, 0\right)\right\} \\
& =\int_{0}^{\infty}\left(1-F_{X-\mu_{X}}(u)\right) d u+\int_{-\infty}^{0} F_{X-\mu_{X}}(u) d u .
\end{aligned}
$$

We have the following result.

THEOREM 5.2. Let $X_{1}$ and $X_{2}$ be two nonnegative random variables with probability density functions $g_{1}$ and $g_{2}$ and corresponding length-biased probability density functions $g_{l_{1}}$ and $g_{l_{2}}$, respectively. Assume $0<E\left|X_{i}\right|<\infty$, and $\mu_{X_{1}}=\mu_{X_{2}}$, then

$$
\Delta_{l_{1}}=\frac{1}{2} \int_{0}^{\infty} \max \left(g_{1}(x), g_{l_{1}}(x)\right) d x \geq \Delta_{l_{2}}=\frac{1}{2} \int_{0}^{\infty} \max \left(g_{2}(x), g_{l_{2}}(x)\right) d x
$$

if and only if

$$
E\left|X_{1}-\mu_{X_{1}}\right| \geq E\left|X_{2}-\mu_{X_{2}}\right|
$$

Let $\left\{F_{\theta}: \theta \in \Theta\right\}$. The survival function $\bar{H}$, given by

$$
\bar{H}(x)=\int_{\Theta} \bar{F}_{\theta}(x) d K(\theta),
$$

is a mixture of $\bar{F}_{\theta}$, where $K$ is the mixing distribution. Assume that $\bar{F}_{\theta}(x)$ has density $f(x)$, then the density of $h$ is given by

$$
h(x)=\int_{\Theta} f_{\theta}(x) d K(\theta) .
$$

A sufficient condition for dilation is that $\mu_{X_{1}}=\mu_{X_{2}}$ and $g_{1}-g_{2}$ has two sign changes, zero disregarded and order of the sign sequence is,,+-+ , this is denoted by $X_{1} \stackrel{\text { dil }}{\leq} X_{2}$, where $g_{1}$ and $g_{2}$ are the probability functions of $X_{1}$ and $X_{2}$, respectively.

THEOREM 5.3. Let $X_{1}$ and $X_{2}$ be two nonnegative random variables with probability density functions $g_{1}$ and $g_{2}$ and corresponding length-biased probability density functions $g_{l_{1}}$ and $g_{l_{2}}$, respectively. Consider the mixtures given by

$$
h_{1}(x)=\int_{\Theta} g_{1 \theta}(x) d K_{1}(\theta), \quad h_{2}(x)=\int_{\Theta} g_{2 \theta}(x) d K(\theta),
$$


respectively. Then

$$
\int_{0}^{\infty}\left|h_{1}(x)-h_{l_{1}}(x)\right| d x \geq \int_{0}^{\infty}\left|h_{2}(x)-h_{l_{2}}(x)\right| d x
$$

if $E\left|X_{1}-\mu_{X_{1}}\right| \geq E\left|X_{2}-\mu_{X_{2}}\right|$ and $K_{1} \stackrel{\text { dil }}{\geq} K_{2}$.

Proof. Note that

$$
\begin{aligned}
\int_{0}^{\infty} \max \left(h(x), h_{l}(x)\right) d x & =\int_{0}^{\infty} \max \left(\int_{\Theta} f_{\theta}(x) d K(\theta), \int_{\Theta} f_{l_{\theta}}(x) d K(\theta)\right) d x \\
& \geq \int_{\Theta}\left(\int_{0}^{\infty} \max \left(f_{\theta}(x), f_{l_{\theta}}(x)\right) d x\right) d K(\theta),
\end{aligned}
$$

where $f_{l_{\theta}}(x)$ is the length-biased probability density function corresponding to $f_{\theta}(x)$, and from Theorem 5.2 the result follows.

ACKNOWLEDGEMENT. The authors wish to thank the referees for constructive criticisms which lead to substantial improvement of the paper.

\section{REFERENCES}

[1] R. E. Barlow, A. W. Marshall, and F. Proschan, Properties of probability distributions with monotone hazard rate, Ann. Math. Statist. 34 (1963), 375-389. MR 30\#1559. Zbl 249.60006 .

[2] M. Brown, Approximating IMRL distributions by exponential distributions, with applications to first passage times, Ann. Probab. 11 (1983), no. 2, 419-427. MR 84h:60038a. Zbl 519.62015.

[3] N. Ebrahimi and F. Pellerey, New partial ordering of survival functions based on the notion of uncertainty, J. Appl. Probab. 32 (1995), no. 1, 202-211. MR 96c:62157. Zbl 817.62082.

[4] R. C. Gupta and O. Akman, Statistical inference based on the length-biased data for the inverse Gaussian distribution, Statistics 31 (1998), no. 4, 325-337. MR 2000e:62012. Zbl 930.62020.

[5] J. Keilson, Markov Chain Models-Rarity and Exponentiality, Applied Mathematical Sciences, vol. 28, Springer-Verlag, New York, 1979. MR 80f:60061. Zbl 411.60068.

[6] M. Zelen and M. Feinleib, On the theory of screening for chronic diseases, Biometrika 56 (1969), 601-614. MR 41\#2871. Zbl 184.23703.

Broderick O. Oluyede: Department of Mathematics and Computer SCience, Georgia SOUTHERN UNIVERSITY, STATESBORO, GA 30460, USA

E-mail address: boluyede@gasou.edu

MekKi Terbeche: Department of Mathematics ANd Computer SCience, GeORgia SOUTHERN UNIVERSITY, STATESBORO, GA 30460, USA 


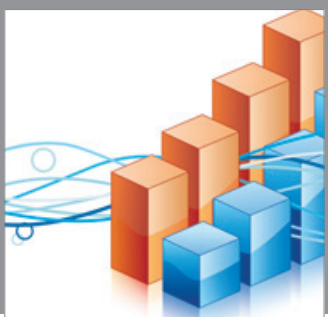

Advances in

Operations Research

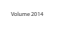

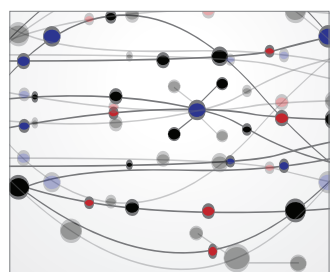

\section{The Scientific} World Journal
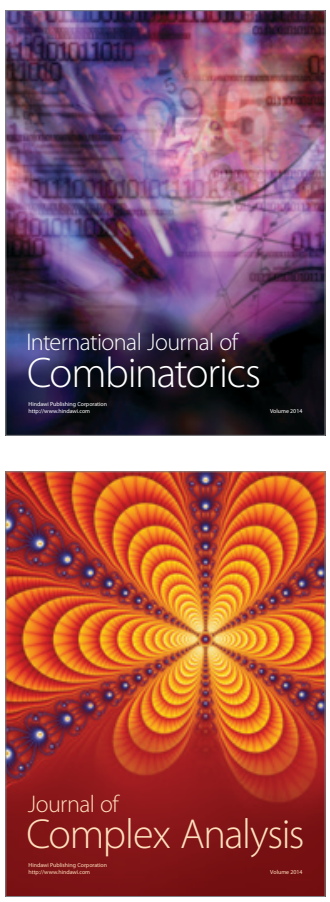

International Journal of

Mathematics and

Mathematical

Sciences
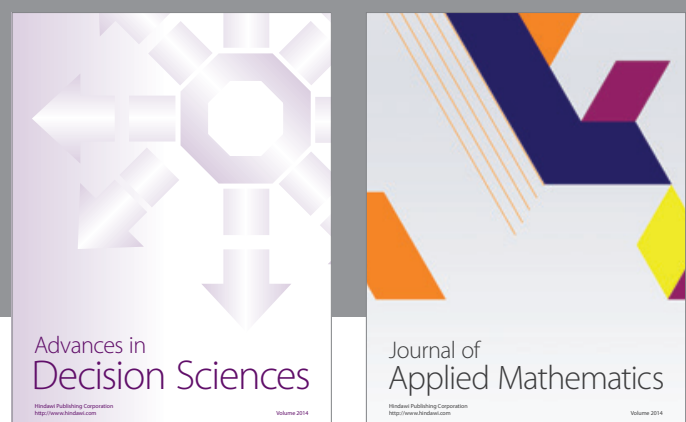

Journal of

Applied Mathematics
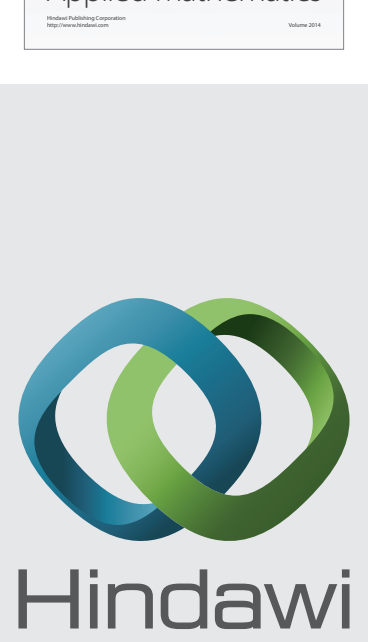

Submit your manuscripts at http://www.hindawi.com
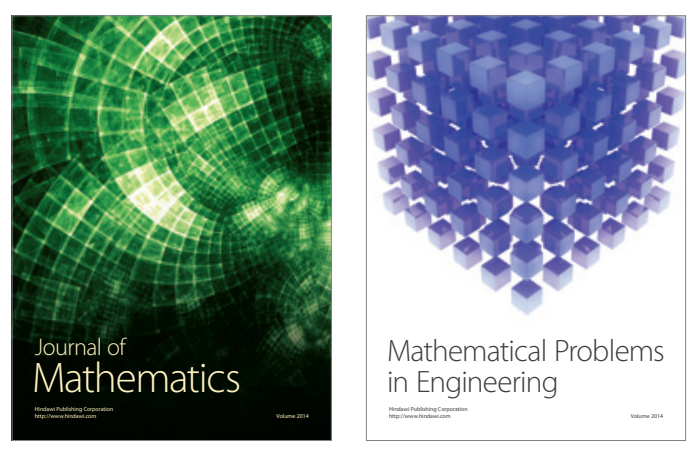

Mathematical Problems in Engineering
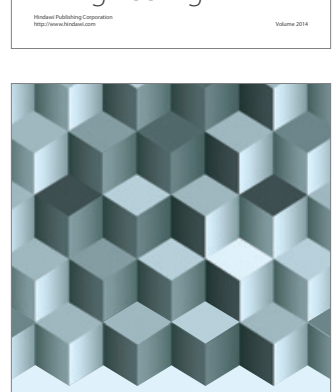

Journal of

Function Spaces
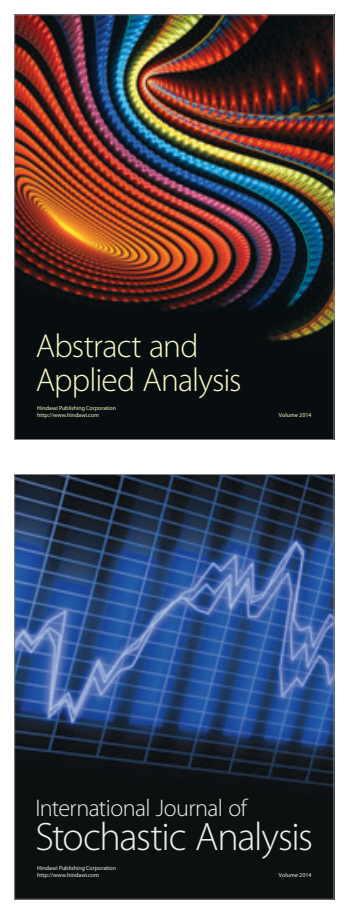

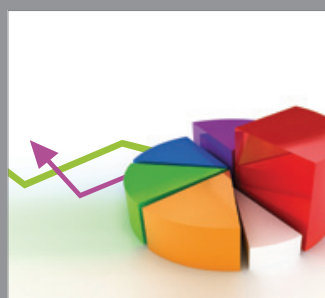

ournal of

Probability and Statistics

Promensencen
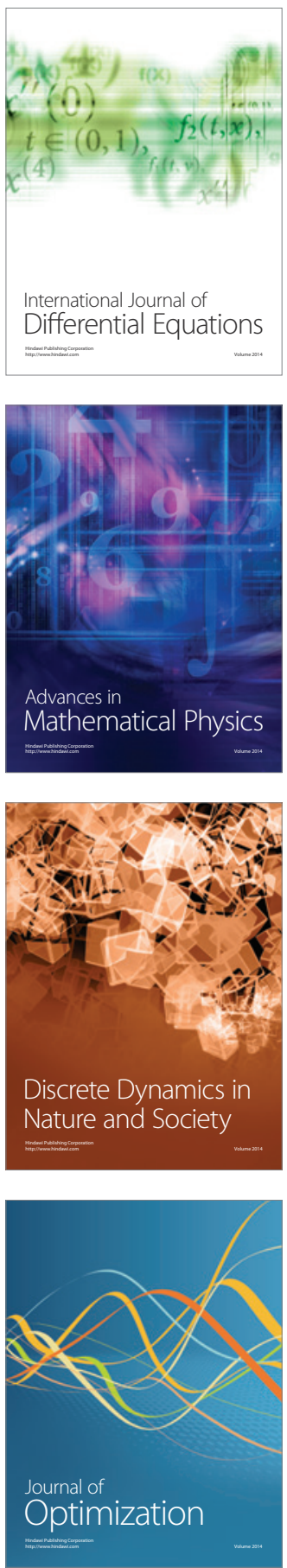